\title{
NUCLEATION AND GROWTH OF PRECIPITATES IN DEMIXING GLASS MELTS IN THE SYSTEM Na ${ }_{2} \mathrm{O}-\mathrm{CaO}-\mathrm{SiO}_{2}$
}

\author{
Klaus HUNTEBRINKER and Ulrich WÜRZ \\ Max-Planck-Institut für Biophysikalische Chemie, Postfach 2841, D-3400 Göttingen I, Fed. Rep. Germany \\ Günther H. FRISCHAT \\ Institut für Nichtmetallische Werkstoffe, Technische Universität Clausthal, Zehntnerstr. 2a, D-3392 Clausthal-Zellerfeld, \\ Fed. Rep. Germany
}

Received 30 March 1989

Revised manuscript received 29 June 1989

\begin{abstract}
As an example of a first order phase transition with conserved order parameter the demixing of glass-forming quasibinary systems has been investigated by in situ measurement of light scattering during heat treatment. The relative supercooling is consistent with theoretical expectations. Using a two stage heat treatment, nucleation and growth are decoupled. Nuclei formed at a nucleation temperature $T_{\mathrm{n}}$ can grow to sizes detectable by light scattering at a higher temperature $T_{\mathrm{gr}}$, where no more nuclei are formed. Reducing the nucleation time, one at last ends up with an incubation time, the temperature dependence of which is described by an Arrhenius ansatz. For low particle densities, the growth mechanism can be deduced from the initial increase of the scattering intensity. The proportionality of the latter to $t^{5}$ is characteristic for diffusion controlled growth of spheres in a depleted zone. From extrema in the scattering a definite size is assigned to the aggregate. Then, the number of particles can be deduced from the absolute intensity. For the systems investigated, however, growth time is limited by the occurrence of crystallisation, thus allowing only the determination of relative nucleation rates.
\end{abstract}

\section{Introduction}

In the last few years, there has been growing interest in the demixing of glass forming systems [1]. On the other hand, technical aspects are preponderant in these investigations, as e.g. in the production of opalescent glasses, or in using the precipitates as a first stage for ceramization. On the other hand, quasibinary demixing glass systems are systems, where aspects of a broader scientific interest can be studied in an especially simple manner [2]. The investigations of first order phase transitions are often impeded by effects, which are coupled with the quantities of interest: condensation of gases and the demixing of liquids are influenced by convection. Precipitation in solids may be affected by mechanical strain energy effects, by anisotropy of interfacial energies and by structural singularities such as dislocations and grain boundaries. Additional problems may arise from insufficient transport of heat or inconvenient time scales [3]. In glass forming systems these difficulties often are negligible. Moreover, the transparency of many glasses for visible light is a special experimental advantage as compared to the much studied metallic systems.

\section{Basic concepts}

Thus, light scattering in some glass-forming systems presents itself as an appropriate means to study the progress of first order phase transitions via nucleation and growth [4]. Most experiments, however, are not capable of separating nucleation from growth processes, which complicates interpretation. Therefore, experiments analogous to the 


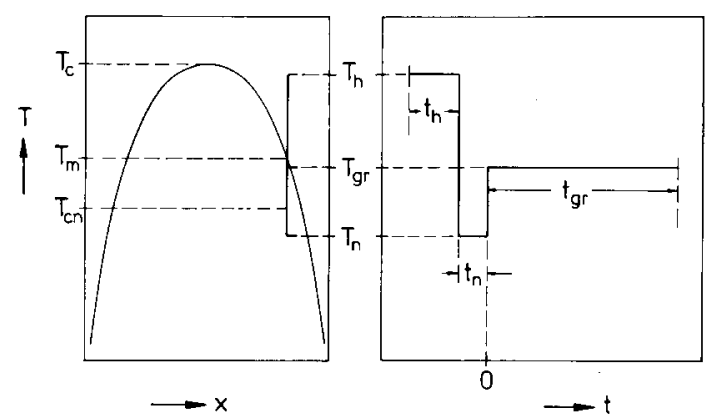

Fig. 1. Miscibility gap with critical temperature $T_{\mathrm{c}}$ (left) and its relation to a typical nucleation and growth experiment (right). The sample is homogenized at temperature $T_{\mathrm{h}}$ for the time $t_{\mathrm{h}}$. Then it is cooled down quickly to the nucleation temperature $T_{\mathrm{n}}$, passing the binodal at $T_{\mathrm{m}}$, and the critical nucleation temperature $T_{\mathrm{cn}}$, and held there for the time $t_{\mathrm{n}}$. Growth time $t_{\mathrm{gr}}$ counts from a quick heating to the growth temperature $T_{\mathrm{gr}}$.

nucleation experiments in condensing vapours by Wagner and Strey [5] are especially interesting.

For the case of demixing systems, the typical schedule of such an experiment is depicted in fig. 1: after homogenization above the binodal, the sample is quickly cooled down to a temperature $T_{\mathrm{n}}$ within the miscibility gap, where nuclei are formed. After a suitable nucleation time $t_{\mathrm{n}}$, the temperature is raised to a temperature $T_{\mathrm{gr}}$, where no more nuclei can be generated, but where existing particles still can grow until they become detectable by light scattering. If they reach a size comparable to or larger than the wavelength of light, deviations from Rayleigh scattering occur, and the radius of spherical particles can be deduced simply from the form of the scattering curve, and the number from the absolute intensity.

A major advantage of this in situ procedure is that no preparation artifacts are introduced. Moreover, if the sample can be homogenized again, a new experiment may be started with the same specimen, thus increasing both economy and reproducibility. However, the number of nuclei must be much lower than in most other experiments, since there should remain enough space for every nucleus to grow to a detectable size without being influenced by neighbouring particles.

\section{Theory}

\subsection{Light scattering}

Far from the critical and spinodal regions, we may assume radially symmetric particles because of the isotropy of the glass matrix. The precipitate can be supposed to be a homogeneous sphere of material with concentration $c_{0}$ corresponding to one flank of the miscibility gap (e.g. almost pure silica glass). The light scattering of such spheres is dealt with in great detail in the literature $[6,7]$ in different approximations, the exact solution is given by Mie [8] as a series expansion.

In contrast to the simple situation in condensing gases, where the light scattering of the matrix phase can be neglected entirely, the contribution of the surroundings of the sphere depends on the growth conditions: the matrix remains homogeneous (though its composition may change) for reaction determined growth, for heat conduction controlled growth, and when diffusion zones strongly overlap (i.e. when the stage of Ostwald ripening is reached). In all these cases, the theory for the scattering of homogeneous spheres suffices.

\subsection{Diffusion zone}

In the case in which we are interested, however, a different mechanism is expected to be dominant: if the demixing process is diffusion controlled, a depleted zone around each particle must be taken into account. In this case the light scattering is influenced or even dominated by the interference between the sphere and its surroundings. We assume the concentrations in the particle, $c_{0}$, and at the border of the particle, $c_{1}$, to be identical with the concentrations defining the miscibility gap. With these boundary conditions, the concentration field can be computed numerically along the lines given by Frank [9] and by Shetterly and Stubler [10]. Further assumption of a linear dependence between refractive index and concentration $[10,11]$ yields the refractive index profile. For the systems studied here, the relative refraction index is always within a few per cent of 1 , thus the Rayleigh-Debye expressions for light scattering are good approximations of the true scattering. 


\subsection{Anomalous scattering}

Goldstein [12] has calculated the light scattering of such an aggregate, treating the sphere in the Rayleigh approximation, and the depletion zone in the Rayleigh-Debye approximation. Hammel and Ohlberg [13] and Shetterley and Stubler [10] have used the latter approximation throughout, and so have we. Unfortunately, it is difficult to improve these calculations of the light scattering amplitude of the depleted zone. However, Mie scattering of a sphere with surrounding depleted shell of constant concentration can be calculated for comparison.

The contributions of sphere and depletion zone to the scattering amplitude cancel exactly in the forward direction (scattering angle $\theta=0^{\circ}$ ). This is easily understood, since at $\theta=0^{\circ}$ the scattering intensity is proportional to the integral of the refractive index over the entire aggregate. This integral vanishes, if the formation of the aggregate is not accompanied by a refractive index defect. For small scattering angles, the intensity rises from zero as $\theta^{4}$, leading to predominant backscattering ("anomalous" scattering [4,12]) as

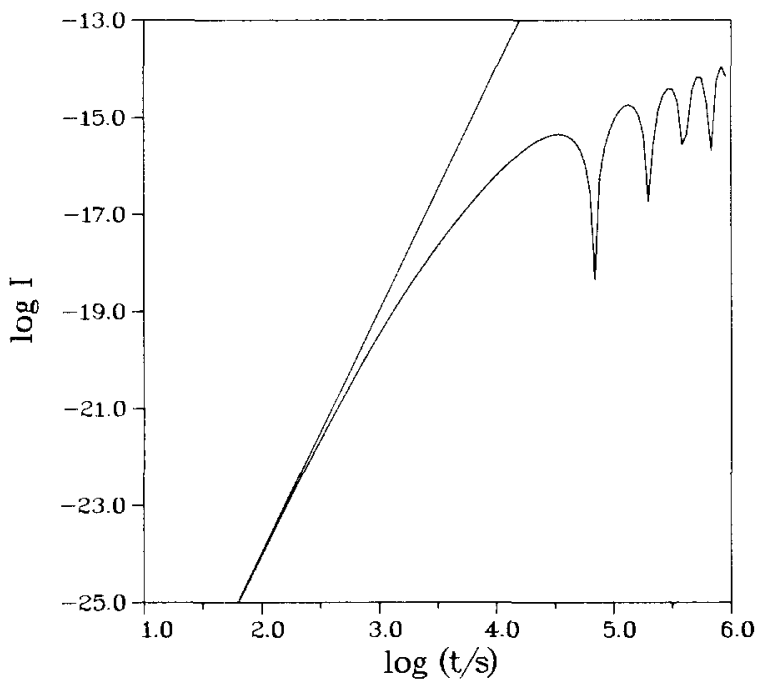

long as the aggregates are small compared to the wavelength of light.

Since we will mainly use the light scattering intensity at $\theta=90^{\circ}$ to monitor the growth of the particles, we calculate $I\left(90^{\circ}, t\right)$ for the aggregate under the conditions of the experiment and plot it in a double logarithmic plot versus time. A value of $5 \times 10^{-14} \mathrm{~cm}^{2} / \mathrm{s}$ [14] was assumed for the diffusion coefficient. The intensities which result from the sphere and from the depleted zone alone are plotted for comparison. These curves coincide for low $t$, showing the expected $t^{3}$-behaviour (which corresponds to the $r^{6}$-dependence of Rayleigh scattering).

Since the Rayleigh scattering amplitudes of the sphere and depleted zone cancel exactly, the initial rise in intensity is proportional to $r^{10}$. Together with the dependence $r \propto \sqrt{t}$ [9] this leads to a rise $\propto t^{5}$ (as already found by Goldstein [12], see fig. 2a). After this very fast increase in intensity, the contribution of the diffusion zone begins to lag behind that of the sphere (fig. 2b), which at last becomes dominant. The later stages roughly resemble those of the sphere alone with its typical minima and maxima, although the intensities are

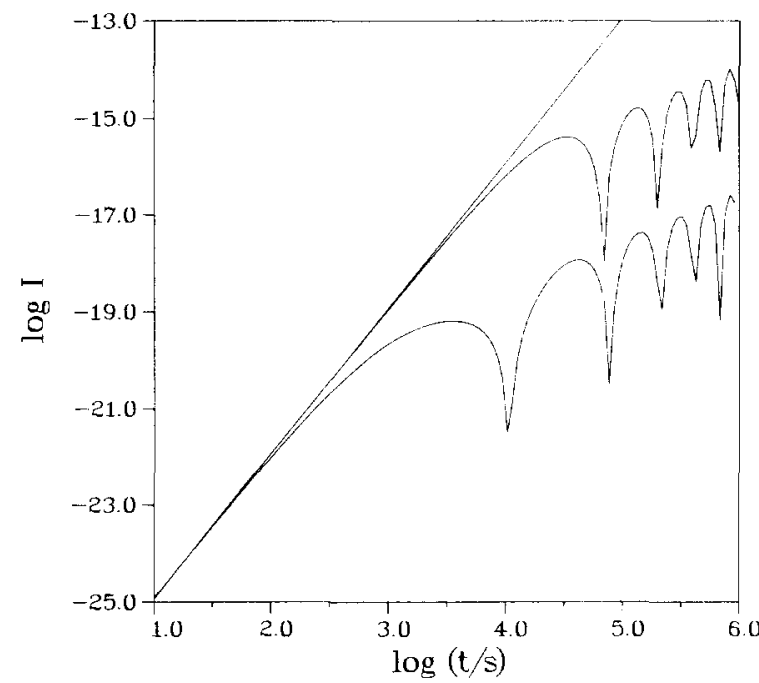

Fig. 2. (a) Light scattering intensity $I\left(90^{\circ}, t\right)$ of a spherical particle growing diffusion-controlled in a depletion zone (no refraction index defect) in the Rayleigh-Debye approximation. The limiting behaviour is indicated by the straight line with slope 5 . The resolution of the graphs (especially of the minima) is limited by the increase of growth time in $10 \%$ increments. (b) Light scattering intensity $I\left(90^{\circ}, t\right)$ for diffusion-controlled growth of the sphere alone (upper curve), and of the depletion zone alone (lower curve) in the Rayleigh-Debye-approximation. The limiting behaviour according to Rayleigh is indicated by the straight line with slope 3 . 


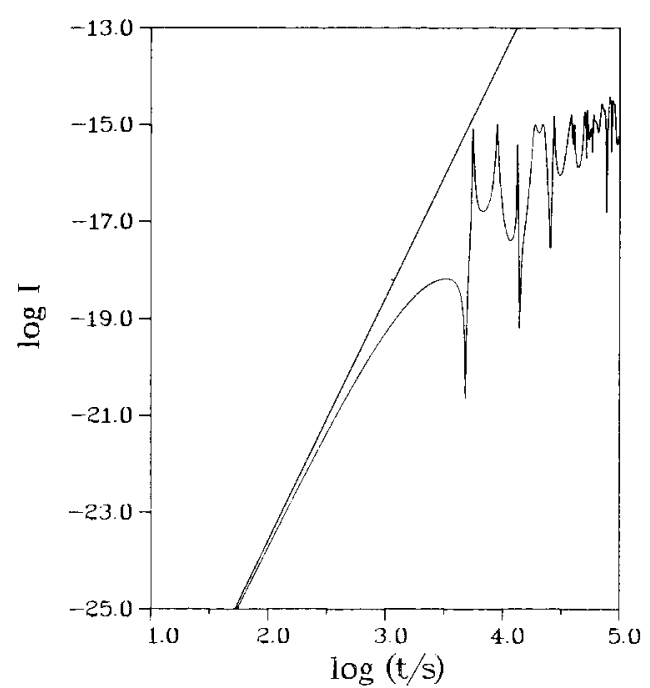

Fig. 3. Light scattering intensity $I\left(90^{\circ}, t\right)$ of a sphere surrounded by a homogeneously depleted shell. The refractive index profile is chosen such that the integral over the refractive index vanishes. Resolution is limited by $1 \%$ increments in $t$.

lower and the extrema shifted. As an estimate of the influence of the degree of approximation in the presented results, the Mie scattering of a homogeneous sphere, surrounded by a homogeneous shell (again with no refractive index defect) is shown in fig. 3. It is plausible from this picture (and from the calculation of contributions of variations of refractive index near the surface of the sphere [15] that noticeable deviations from the intensity given in fig. 2 do not arise much earlier than when the first maximum of the intensity in fig. 2 is reached. A refractive index defect would result in an incomplete cancellation of the leading terms, and hence in a $r^{6}\left(t^{3}\right)$ dependence for small particles.

\subsection{Critical supercooling}

For the flank of the miscibility gap, all nucleation theories predict a sharply defined temperature $T_{\mathrm{cm}}$, where nucleation sets in [16]. With this temperature, the critical supercooling

$x=\left(T_{\mathrm{m}}-T_{\mathrm{cn}}\right) /\left(T_{\mathrm{c}}-T_{\mathrm{m}}\right)$

is defined (see fig. 1 for the definition of the different temperatures). The relevant quantity for comparison with theory is the relative supercooling $y=x_{\mathrm{c}} / x_{0} . x_{0}$ is related to the critical amplitudes (eq. 10.2 to 10.7 in ref. [16]), which are not known for our system. Experimentally, $x_{0}$ is of order unity for condensing gases $\left(x_{0}=1.24 \pm 0.1\right.$ for $\left.\mathrm{CO}_{2}\right)$ and for binary demixing liquids $\left(x_{0}=\right.$ $1.30 \pm 0.1$ for $\left.\mathrm{C}_{7} \mathrm{H}_{14}-\mathrm{C}_{7} \mathrm{H}_{14}\right)$. The remarkable agreement of the two values cited is apparently not accidental.

Following Stauffer et al. [17] one may form the quantities

$X \equiv \tau^{2} \alpha C \xi^{3} / k_{\mathrm{b}}$

and

$Y \equiv\left(\sigma / k_{\mathrm{b}} T_{\mathrm{c}}\right) /\left(\tau^{2} \alpha C / k_{\mathrm{b}}\right)^{2 / 3}$

with $\tau=\left(T-T_{\mathrm{c}}\right) / T_{\mathrm{c}}, \alpha$ the critical exponent of the singular part of the specific heat, $C=A \tau^{-\alpha}, \xi$ the correlation length, $\sigma$ the interfacial tension, and $k_{\mathrm{b}}$ Boltzmann's constant.

Assuming two-scale universality [17] $X$ and $Y$ have the same numerical constants for all systems belonging to the same universality class. On the other hand, according to Langer and Schwartz (eq. 2.18 in ref. [18]) $x_{0}$ can (at least for a " $\phi^{4}$ " theory) be written as

$x_{0}^{2}=16 \sigma \xi^{2} /\left(k_{\mathrm{b}} T_{\mathrm{c}}\right)$

and hence may be expressed in terms of $X$ and $Y$ :

$x_{0}=2 X^{1 / 3} Y^{1 / 2}$.

Thus, $x_{0}$ is expected to be universal, also. Unfortunately, an exact number for $Y$ is lacking for the universality class of the 3-dimensional Ising model to which our system should belong [19]. We thus adopt the experimental value of 1.3 for $x_{0}$ cited above.

\section{Materials and preparation}

The samples were prepared from alkali carbonates, alkaline earth carbonates (p.a. quality, $>99 \%$, from Merck) and high purity $\mathrm{SiO}_{2}$ powder (from Heraeus). Enough powder to produce $150 \mathrm{~g}$ of glass was mixed thoroughly overnight on a roller bank, molten at $1400^{\circ} \mathrm{C}$ for $2 \mathrm{~h}$ in a $\mathrm{Pt}-\mathrm{Au} 5$ crucible, and cooled down to room temperature. 
The crucible was then transferred to a special furnace and heated again to $1400^{\circ} \mathrm{C}$. After 30 min, the melt was stirred with a PtRh stirrer at a rate of about $25 \mathrm{rpm}$ for $2 \mathrm{~h}$. To remove air bubbles, the melt was held for an additional $12 \mathrm{~h}$ at $1400^{\circ} \mathrm{C}$ without stirring, and then cooled down in air.

The glass blocks produced in this manner were free of bubbles or flaws, no sedimentation effects could be detected. To remove mechanical stress, they were heated to the glass transition temperature, held there for $2 \mathrm{~h}$ and cooled down slowly at a rate of $0.5 \mathrm{~K} / \mathrm{min}$ to room temperature.

Glass cylinders of slightly less than $10 \mathrm{~mm}$ diameter were prepared from these blocks with a hollow diamond borer. A facet was cut at their mantle and polished to provide an undisturbed exit for the light scattered at $90^{\circ}$. Then the cylinders were cut into slices of $3 \mathrm{~mm}$ thickness and the upper and lower ends discarded. The plane surfaces of the samples were ground, polished and rinsed with water-free methanol.

\section{Apparatus}

The apparatus used is described elsewhere [20]. The furnace with regulator has the capability for heating and cooling rates of the order of several $\mathrm{K} / \mathrm{s}$ and for keeping the temperature of the sample constant to within about $0.1 \mathrm{~K}$ for several days (up to $900^{\circ} \mathrm{C}$ ). A $4.5 \mathrm{~mW} \mathrm{He}-\mathrm{Ne}$ laser was the source of linearly polarized light, and the scattered light was chopped at a frequency of about $75 \mathrm{~Hz}$ and detected at $90^{\circ}$ with a sensitivity of about $10^{-8}$ of the primary intensity, by use of photo-diodes and lock-in amplifiers.

\section{Results}

\subsection{Temperature-time-transition diagrams}

The first experiments on the system $\mathrm{Li}_{2} \mathrm{O}-\mathrm{SiO}_{2}$ yielded poorly reproducible results. By applying a temperature program according to fig. 1 (the homogeneous samples, prepared as described above, were, however, directly heated to the nucleation temperature) and monitoring the light scattering, highly structured $I\left(90^{\circ}, t\right)$-curves resulted. In some cases, the scattering curves could be related to the expected Mie scattering. Inspection by SEM and optical microscopy showed that surface crystallisation and crystallisation starting from the $\mathrm{SiO}_{2}$-rich precipitates formed by demixing complicated measurement and interpretation.

In order to get an overview of the kinetics of the transition from the homogeneous state, the samples were heated to different temperatures while monitoring the light scattering intensities. After some time the intensity started to increase rapidly. In fig. 4 the time required for a doubling of the scattered intensity is plotted versus temperature.

If demixing is the only reaction, the transition times would become increasingly shorter with increasing temperature, due to higher diffusion rates and increasing nucleation rates, until the critical supersaturation temperature $T_{\mathrm{nc}}$ is crossed. For temperatures higher than $T_{\mathrm{nc}}$, nuclei can no longer be formed, and hence the transformation times should tend to infinity. However, the outcome of this experiment and of the preliminary ones mentioned above demonstrates that the glassy state is a metastable state. In fig. 4 the two C-shaped curves are superimposed, the upper curve is associated with crystallization. Demixing and crystal-

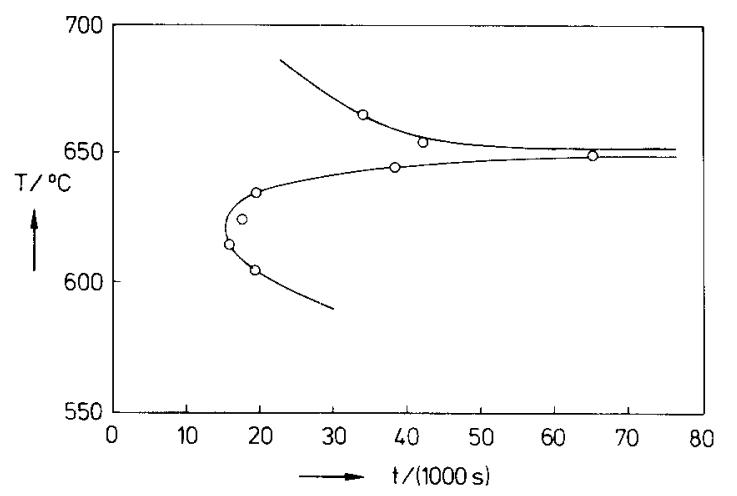

Fig. 4. TTT-diagram for a $13.1 \mathrm{~mol} \% \mathrm{Na}_{2} \mathrm{O}-11 \mathrm{~mol} \% \mathrm{CaO}-75.9$ mol\% $\mathrm{SiO}_{2}$ sample. The time needed for the doubling of the scattering intensity is plotted as a measure for the progress of the transformation. Two processes can be distinguished: the lower C-shaped curve is due to demixing, the upper one is associated with crystallisation. 
lisation can be studied separately as long as these two curves do not overlap. Temperature-timetransition (TTT)-plots and DSC measurements for different sample compositions in the $\mathrm{Na}_{2} \mathrm{O}-\mathrm{CaO}$ $-\mathrm{SiO}_{2}$ system show that the temperature at which the maximum crystallisation rate occurs, is only slightly dependent on the composition of the samples. The temperature of demixing, however, varies strongly with the $\mathrm{CaO}$ content, following the lines of the "master curve" of Kawamoto and Tomozawa [21]. Unfortunately, we did not find a system which demixed in a reasonable time period and could be rehomogenized directly in the apparatus.

\subsection{Critical supercooling}

According to the TTT-plots, there exists a relatively well-defined temperature $T_{\mathrm{cm}}$ where nucleation begins. For a $13.1 \mathrm{~mol} \% \quad \mathrm{Na}_{2} \mathrm{O}-11 \mathrm{~mol} \%$ $\mathrm{CaO}-75.9 \mathrm{~mol} \% \mathrm{SiO}_{2}$ sample, with $T_{\mathrm{c}}=1003^{\circ} \mathrm{C}$ the binodal temperature is $T_{\mathrm{m}}=702^{\circ} \mathrm{C}$ [21], the critical supercooling $x=\left(T_{\mathrm{m}}-T_{\mathrm{cn}}\right) /\left(T_{\mathrm{c}}-T_{\mathrm{m}}\right)=$ 0.17 , which results in a relative supercooling of 0.13 , which compares favourable with the value for the classical limit as deduced from fig. 9 of ref. [18].

\subsection{Diffusion controlled growth}

TTT-diagrams give a rough and relatively quick overview of the progress of the transformation. Since both nucleation and growth of precipitates and/or crystals lead to an increase of the scattered intensity, quantitative interpretation is difficult. However, fig. 4 shows that for a $13.1 \mathrm{~mol} \%$ $\mathrm{Na}_{2} \mathrm{O}-11 \mathrm{~mol} \% \mathrm{CaO}-75.9 \mathrm{~mol} \% \mathrm{SiO}_{2}$ sample at $650^{\circ} \mathrm{C}$ there are about $10^{5} \mathrm{~s}$, where the growth of nuclei formed at lower temperatures can be followed without being disturbed by crystallization (which in this case starts solely from the sample surface).

Fig. 5 shows the increase of scattering intensity $I$ with growth time $\left(t_{\mathrm{gr}}\right)$ at $650^{\circ} \mathrm{C}$ for such a sample with different nucleation periods $\left(t_{\mathrm{n}}\right)$ at $615^{\circ} \mathrm{C}$ in a double logarithmic plot. For the 120 min nucleation period, the value of the slope of the resulting straight line is about 3 as can be seen

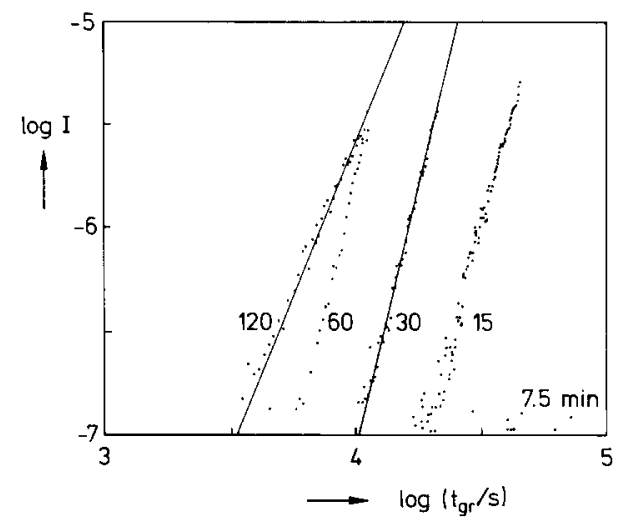

Fig. 5. Experimental light scattering intensities $I\left(90^{\circ}, t\right)$ as a function of growth time $t_{\mathrm{gr}}$. Sample composition is $13.1 \mathrm{~mol} \%$ $\mathrm{Na}_{2} \mathrm{O}-11 \mathrm{~mol} \% \mathrm{CaO}-75.9 \mathrm{~mol} \% \mathrm{SiO}_{2}$. Growth temperature $T_{\mathrm{gr}}=650^{\circ} \mathrm{C}$. Nucleation temperature $T_{\mathrm{n}}=615^{\circ} \mathrm{C}$. Nucleation times $t_{\mathrm{n}}$ are indicated. The guide lines have slopes of 3 and 5 , respectively.

from comparison with the left guide line in fig. 5 . This slope value is consistent with the notion of Rayleigh scattering of spherical particles $\left(I \propto r^{6}\right)$ [6] growing diffusion-controlled $\left(r \propto t^{1 / 2}\right)$ [9] in a homogeneous matrix, a situation which is to be expected when diffusion zones strongly overlap.

Nucleation periods of 60,30 , and $15 \mathrm{~min}$, and thus lower particle numbers, result in parallel, essentially equidistant straight lines in fig. 5 with a slope of 5, (as can be seen from comparison with the right auxiliary line). This result is consistent with diffusion controlled growth, as discussed above (see fig. 2a). Within $7.5 \mathrm{~min}$, essentially no nuclei have been formed, leading to the conclusion that the time to reach local thermodynamic equilibrium, and thus the time to reach stationary nucleation conditions is of the same order of magnitude. This incubation time will be discussed below.

In situations where the assumption of independent growth of particles is best fulfilled, as in fig. 6 , a slope of $5.0 \pm 0.2$ is found.

The $t^{5}$ dependence of the scattered intensity definitely excludes some mechanisms; reaction controlled growth $(r \propto t)$ would show a slope of 6 [23], diffusion controlled growth $\left(r \propto t^{1 / 2}\right)$ in a homogeneous depleted matrix [9] (as might occur for strongly overlapping diffusion zones) would 


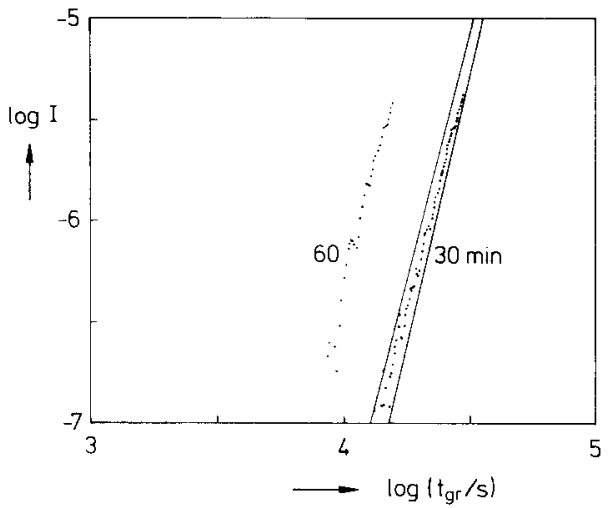

Fig. 6. Experimental light scattering intensities $I\left(90^{\circ}, t\right)$ as a function of growth time $t_{\mathrm{gr}}$. Sample composition is $13.1 \mathrm{~mol} \%$ $\mathrm{Na}_{2} \mathrm{O}-11 \mathrm{~mol} \% \mathrm{CaO}-75.9 \mathrm{~mol} \% \mathrm{SiO}_{2}$. Growth temperature $T_{\mathrm{gr}}=650^{\circ} \mathrm{C}$. Nucleation temperature $T_{\mathrm{n}}=605^{\circ} \mathrm{C}$. Nucleation times $t_{\mathrm{n}}$ are indicated. The guide lines have slopes of 4.8 and 5.2 , respectively.

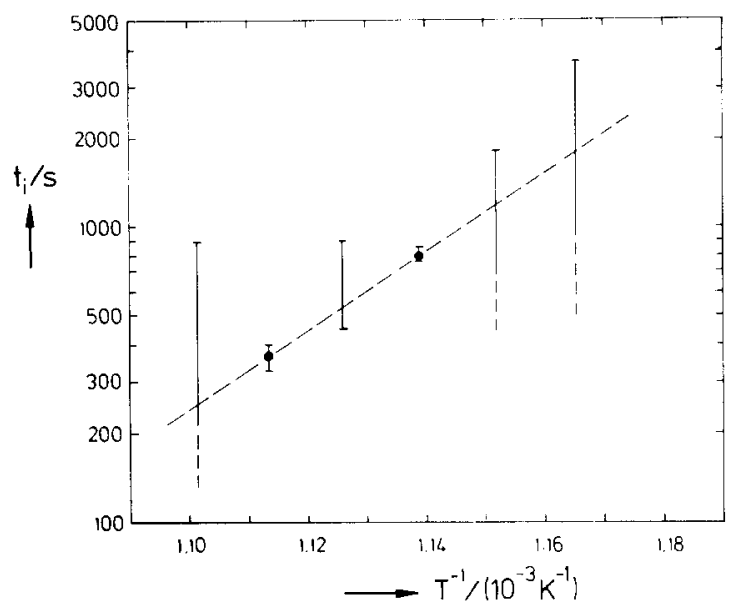

Fig. 8. Incubation times as deduced from equidistance in $\log I$ versus $\log \left(t_{\mathrm{n}}-t_{\mathrm{i}}\right)$ plots

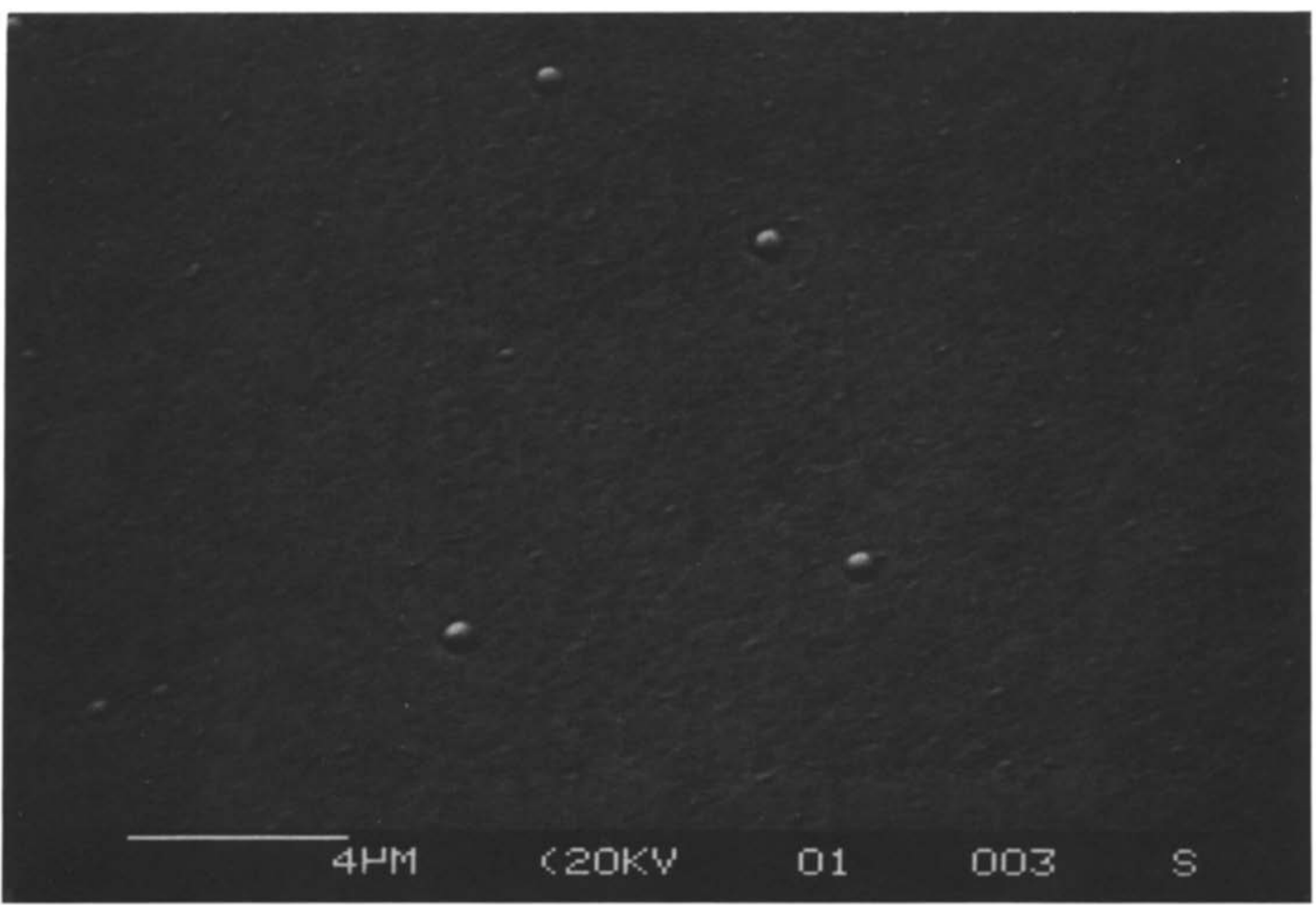

Fig. 7. Scanning electron microscopy picture. Sample composition is $13.1 \mathrm{~mol} \% \mathrm{Na}_{2} \mathrm{O}-11 \mathrm{~mol} \% \mathrm{CaO}-75.9 \mathrm{~mol} \% \mathrm{SiO}_{2} . \mathrm{Nucleation}$ time $t_{\mathrm{n}}=30 \mathrm{~min}$ at nucleation temperature $T_{\mathrm{n}}=595^{\circ} \mathrm{C}$. Growth time $t_{\mathrm{gr}}=20 \mathrm{~h}$ at growth temperature $T_{\mathrm{gr}}=650^{\circ} \mathrm{C}$. The sample was etched in $2 \%$ HF for $40 \mathrm{~s}$ and sputtered with gold. The spherical precipitates have equal size and are widely separated. For the scattering intensity see fig. 9 . 
lead to a slope of 3 as discussed, and for Ostwald ripening [24] $\left(r \propto t^{1 / 3}\right)$ the slope should be 2 (for Rayleigh scatterers at least). Thus, we conclude that the growth of the particles is indeed diffusion controlled. In fact, scanning electron microscopy of the sample for which data is given in fig. 7 reveals widely separated particles of equal size in accordance with the assumptions of the theory.

\subsection{Incubation times}

The ordinate distances between the straight lines in fig. 5 contain information about the nucleation rate, but also about the growth conditions during both nucleation and growth periods. If there exists an incubation time, $t_{\mathrm{i}}$, for the nucleation, one would expect from an otherwise stationary nucleation equal spacings in a $\log I$ versus $\log \left(t_{\mathrm{n}}-t_{\mathrm{i}}\right)$ diagram. From this requirement, a value for $t_{\mathrm{i}}$ can be estimated for each $T_{n}$. The temperature dependence of these times can be deduced from fig. 8 and is consistent with an Arrhenius ansatz. The value $(260 \pm 60) \mathrm{kJ} / \mathrm{mol}$ for the activation energy, however, is lower than expected from diffusion data [14,22], which may be attributed to the fact that diffusion starts from a quenched state.

\subsection{Mie scattering}

Considering the experiments of Wagner and Strey [5], it would be appropriate to allow growing of the precipitates to sizes where Mie-maxima occur in the scattering curves. However, the maxima occurring in figs. 2 or 3 are not present in the experimental curves of fig. 5 even for the longest possible times. This is consistent with the particles in fig. 7 , the size of which is only close to showing the first maximum in scattering. However, looking more closely at figs. 5, 6 and 9 one indeed can see a difference between the experimental curves and the straight lines which becomes more and more pronounced, as the times involved become larger. These deviations are reminiscent of the ripples which frequently occur in Mie scattering curves [7] and contain relevant information about the size of the scatterer. Unfortunately, as mentioned earlier, the theoretical scattering curves of a sphere plus diffusion zone do not exist in a sufficient ap-

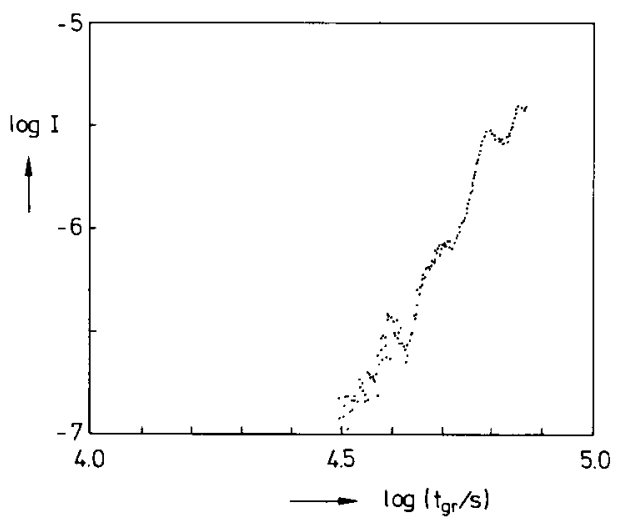

Fig. 9. Experimental light scattering intensity $I\left(90^{\circ}, t\right)$ as a function of growth time $t_{\mathrm{gr}}$. The sample is the one shown in fig. 7 .

proximation to allow discussion of the ripple structure. As opposed to most structures that can be treated exactly (compare fig. 3), the ripples in our case seem to be essentially equidistant in log $t_{\mathrm{g}}$. One possible origin of the ripples could be a strain field around the precipitate, giving rise to a refractive index with tensor properties.

\subsection{Ostwald ripening}

Another strategy to increase the size of the particles and reach the region of Mie scattering is to increase the annealing times at higher supersaturation (lower temperatures). However, not only the growth of particles is enhanced due to higher supersaturation, but also the nucleation rate. The density of particles becomes so high that they cannot grow independently over a longer period of time, and instead, diffusion zones begin to overlap until Ostwald ripening becomes the dominant mechanism. Therefore, the curves in fig. 10 do not show the curvature expected from comparison with the theoretical curves of fig. 2 , but level off due to the exhaustion of the matrix. After a plateau, the light scattering increases again due to growth of the large particles at the expense of the small ones (Ostwald ripening). For Rayleigh scatterers, the height of the plateau is proportional to $N_{i} \cdot r_{i}^{6}$. Since the precipitated volume (and hence $N_{i} \cdot r_{i}^{3}$ ) 


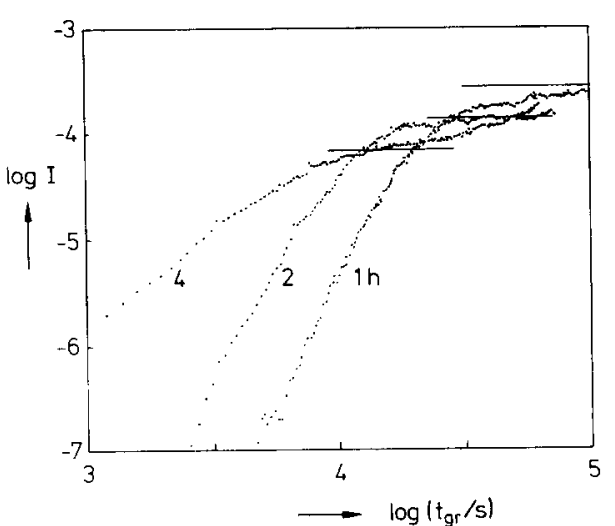

Fig. 10. Experimental light scattering intensity $I\left(90^{\circ}, t\right)$ as a function of growth time $t_{\mathrm{gr}}$. Sample composition is $13.1 \mathrm{~mol} \%$ $\mathrm{Na}_{2} \mathrm{O}-11 \mathrm{~mol} \% \mathrm{CaO}-75.9 \mathrm{~mol} \% \mathrm{SiO}_{2}$. Growth temperature $T_{\mathrm{gr}}=650^{\circ} \mathrm{C}$. Nucleation temperature $T_{\mathrm{n}}=615^{\circ} \mathrm{C}$. Nucleation times $t_{\mathrm{n}}$ are indicated. Before the start of Ostwald ripening plateaus show up in the intensities due to exhaustion of the matrix. Their expected relative heights are indicated. After the plateau a slope value of 2 is expected for later times. The beginning of the time scale is poorly determined because particle growth is already appreciable during the long nucleation times.

should be the same for the three curves of fig. 10 when the plateau is reached,

$r_{1}^{3}: r_{2}^{3}: r_{3}^{3}=1: 2: 4$

for

$N_{1}: N_{2}: N_{3}=4: 2: 1$.

Thus, the plateau values should increase by a factor of 2 for decreasing nucleation times, as indicated in the figure.

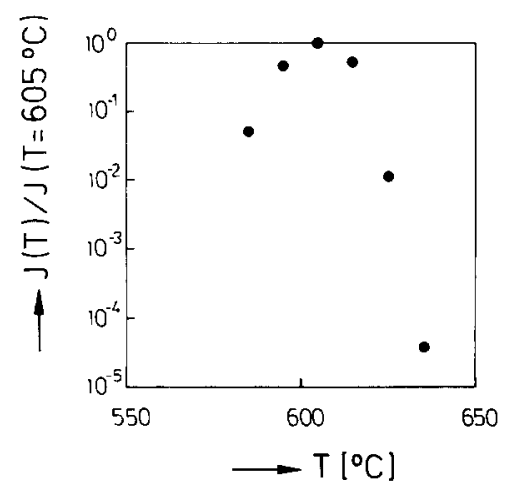

Fig. 11. Relative nucleation rates. The errors can be estimated to lie within a factor of 2

\subsection{Relative nucleation rates}

As is evident from sections 6.4 to 6.6 , it was impossible to reach the Mie-scattering region for the samples which were investigated most thoroughly. Hence, it was impossible to determine both the size and the number of the precipitates. However, when comparing the scattering intensities of samples with identical growth conditions, but different nucleation temperatures, information on the relative nucleation rates can be deduced. They are depicted in fig. 11.

\section{Conclusions}

In conclusion, we have shown that in situ measurements of light scattering during heat treatment can give valuable information about the kinetics of first order phase transitions in glass forming systems. From the shape of temperature-time-transition diagrams it can be deduced, whether one or more phase transitions (e.g. demixing and crystallization) are involved. If the temperature of maximal crystallization velocity is significantly higher than that of demixing, the latter can be studied separately. Then, the value of critical supercooling can be extracted directly from the TTT-diagram. For $13.1 \mathrm{~mol} \% \mathrm{Na}_{2} \mathrm{O}-11 \mathrm{~mol} \%$ $\mathrm{CaO}-75.9 \mathrm{~mol}^{\circ} \mathrm{SiO}_{2}$ the critical supercooling is consistent with theoretical expectations.

Moreover, by a heat treatment in two steps, the nucleation times and temperatures can be varied independently from the growth conditions: nuclei formed at temperature $T_{\mathrm{n}}$ can grow to detectable size at a higher temperature $T_{\mathrm{gr}}$, where no more nuclei can be formed. A temperature dependent incubation time is found for the nucleation process. Thereafter, nucleation is stationary and homogeneous. The growth mechanism can be deduced from the time dependence of the intensity of the scattered light. For low particle densities, diffusion controlled growth of spherical precipitates occurs in a depletion zone as indicated by the initial intensity increase proportional to $t^{5}$. The temperature dependence of the nucleation rates is consistent with classical nucleation theory. 
If the particles can grow to sizes where Mie maxima occur in the scattering curves, absolute values for the nucleation rates can be obtained. In the case of the system investigated most thoroughly, the measurement of later growth stages was disturbed by surface crystallisation. Some fine structure of the scattering curves could not be clearly related to properties of the precipitate.

We thank Professor M. Kahlweit for the opportunity to carry out these investigations in his lab. We gratefully acknowledge many clarifying discussions with $M$. Teubner and the expert technical assistance of $\mathrm{G}$. Wiese and M. Grubic. This work was supported by the SFB 126, GöttingenClausthal.

\section{References}

[1] O.V. Mazurin and E.A. Porai-Koshits, eds., Phase Separation in Glasses (North-Holland, Amsterdam, 1984).

[2] D.R. Uhlmann and B. Chalmers, in: Nucleation Phenomena, D.E. Gushee, ed. (Am. Chem. Soc., Washington, 1966).

[3] K.C. Russell, Adv. Coll. Interf. Sci. 13 (1今80) 205.

[4] N.S. Andreev, J. Non-Cryst. Solids 30 (1978) 99.

[5] P.E. Wagner and R. Strey, J. Phys. Chem. 80 (1984) 5266.
[6] H.C. van de Hulst, Light Scattering by Small Particles (Wiley, New York, 1957).

[7] M. Kerker. The Scattering of Light and other Electromagnetic Radiation (Academic Press, New York, 1969).

[8] G. Mie, Ann. Phys. 25 (1908) 377.

[9] F.C. Frank, Proc. Roy. Soc. London A201 (1950) 586.

[10] D.M. Shetterly and R.J. Stubler, J. Am. Ceram. Soc. 58 (1975) 227

[11] H. Scholze, Glas - Natur, Struktur und Eigenschaften, 2. Auflage (Springer, Berlin, Heidelberg, New York, 1977).

[12] M. Goldstein, J. Appl. Phys. 34 (1963) 1928.

[13] J.J. Hammel and S.M. Ohlberg, J. Appl. Phys. 36 (1965) 1442.

[14] J.J. Hammel, J. Chem. Phys. 46 (1967) 2234.

[15] M. Teubner, private communication.

[16] J.D. Gunton, M. San Miguel and P.D. Sahni, in: Phase Transitions and Critical Phenomena, Vol. 8, C. Domb and J.L. Lebowitz, eds. (Academic Press, London, 1983).

[17] D. Stauffer, M. Ferer and M. Wortis, Phys. Rev. Lett. 29 (1972) 345.

[18] J.S. Langer and A.J. Schwartz, Phys. Rev. A21 (1980) 948.

[19] C. Domb and M.S. Green, eds., Phase Transitions and Critical Phenomena (Academic Press, London, 1983).

[20] K. Huntebrinker, B. Wemheuer, M. Grubic and U. Würz, Rev. Sci. Instr. 60 (1989) 953.

[21] Y. Kawamoto and M. Tomozawa, Phys. Chem. Glasses 22 (1981) 11.

[22] J.J. Hammel, J. Mickey and H.R. Golob, J. Coll. Int. Sci. 27 (1968) 329.

[23] J.W. Mullin, Crystallisation, 2nd ed. (Butterworths, London, 1972).

[24] C. Wagner, Z. Elektrochem. 65 (1961) 243. 